\title{
Autocatalysis and phenotypic expression of Drosophila homeotic gene Deformed: its dependence on polarity and homeotic gene function
}

\author{
ACAIMO GONZÁLEZ-REYES, ANA MACÍAS AND GINÉS MORATA \\ Centro de Biología Molecular, Universidad Autónoma de Madrid, 28049 Madrid, Spain
}

\section{Summary}

Previously published experiments have shown that the endogenous $D f d$ gene can be ectopically activated by its own (heat-shock-driven) product in a subset of cells of different segments. This results in the differentiation of maxillary structures like cirri and mouth hooks in places where they normally do not appear, and represents a phenomenon of autocatalysis of homeotic gene function that differs from the normal activation process. We show that this out-of-context activation occurs in cells belonging to the anterior compartments of the three thoracic and the A1 to A8 abdominal segments and that it requires the normal function of the polarity genes wingless (wg) and engrailed (en). The wg product, in addition to that of $\boldsymbol{D f d}$, appears to be sufficient to activate the endogenous $D f d$ gene in many embryonic

\section{Introduction}

The morphological diversity along the parasegmental trunk of Drosophila is achieved through the function of the homeotic genes (Lewis, 1978; Sánchez-Herrero et al., 1985; Mahaffey and Kaufman, 1988). These are clustered in the Antennapedia (ANT-C) and bithorax (BX-C) complexes, which together contain at least eight homeotic genes (see reviews by Duncan, 1987; Mahaffey and Kaufman, 1988; McGinnis and Krumlauf, 1991). Those of the ANT-C (labial, lb, proboscipedia, pb, Deformed, Dfd, Sex combs reduced, Scr, and Antennapedia, Antp) determine the development of the head and part of the thorax (parasegments 0-5. Martínez-Arias and Lawrence, 1985), and those of the BX-C (Ultrabithorax, Ubx, abdominal-A, abd-A and Abdominal-B, $A b d-B$ ) are responsible for the development of the remaining thorax and the abdomen (parasegments 514). All the homeotic genes encode nuclear proteins with a DNA binding domain, the homeobox (McGinnis et al., 1984; Scott and Weiner, 1984; Gehring, 1987), suggesting that they perform their functions by recognizing and binding to specific DNA sequences of other subsidiary genes whose activities they regulate. Indeed, homeoproteins have been shown to bind to a number of DNA sequences in the genome (Desplan et al., 1985), although the number of sites to which they bind in vivo is not yet known. cells. We have studied the effect of several homeotic genes on $\boldsymbol{D} \boldsymbol{f} d$ activation and phenotypic expression: $\mathrm{Scr}$, $A n t p, U b x$ and $A b d-B$ repress $D f d$ both transcriptionally and at the phenotypic level, if their products are in sufficient amounts. The endogenous $a b d-A$ gene does not have a noticeable effect, but when it is replaced by an hsp70-abd-A gene, which produces a high and uniform level of expression, the phenotypic expression of $D f d$ is suppressed. Our results also suggest that the differentiation of cirri is induced by $D f d$-expressing cells in nonexpressing neighboring cells, and that this interaction occurs across the parasegmental border.

Key words: Drosophila, homeotic genes, phenotypic suppression, Deformed, heat shock.
The homeotic genes also bind to the promoter regions of other homeotics (Beachy et al., 1988; Krasnow et al., 1989), which results in transcriptional regulation between them. The gene Antp is down-regulated by $U b x$ (Carroll et al., 1986; Hafen et al., 1984; Wirz et al., 1986) and in turn $U b x$ is down-regulated by $a b d-A$ (Struhl and White, 1985). Furthermore, some homeoproducts, like those of $U b x$ and $D f d$, bind to their own promoter and autoregulate their own expression (Bienz and Tremml, 1988; Kuziora and McGinnis, 1988a; Müller et al., 1989; Bergson and McGinnis, 1990; Regulski et al., 1991).

There is a peculiar and unexplained fact about the homeotic genes: the position of the ANT-C and BX-C homeotic genes along the chromosome correlates with their pattern of expression along the body (Lewis, 1981; Mahaffey and Kaufman, 1988). A similar correlation has been found for the Hox-C complexes in mouse and humans (Dolle and Duboule, 1989). Moreover, the genes acting more posteriorly in the body suppress phenotypically those acting more anteriorly, e.g., if two homeotic genes are activated together in the same metamere, this will acquire the identity determined by the homeotic gene acting more posteriorly (Struhl, 1983; Busturia and Morata, 1988; González-Reyes and Morata, 1990). A similar observation has also been made in the mouse homeobox genes (Duboule, 1991; Lufkin et al., 1991), where it has been 
called 'posterior prevalence'. Thus, it is a phenomenon of general occurrence, and may reflect a general property of homeotic genes of both insects and vertebrates.

In Drosophila, phenotypic suppression has currently been explained in terms of the phenomenon of down-regulation described above. However, in the case of Antp and $U b x$, it has been shown that the phenotypic suppression of Antp by $U b x$ can take place at the post-transcriptional level (González-Reyes and Morata, 1990; González-Reyes et al., 1990); the coexpression at high levels of both Antp and Ubx proteins results in the identity dictated by $U b x$ alone, that is, the Antp protein is present but made ineffective by the Ubx protein. This indicates that the phenomenon of phenotypic suppression results from interactions between homeotic products that may compete for the same binding sites of downstream genes.

The homeotic gene $D f d$ (Chadwick and McGinnis, 1987; Regulski et al., 1987; reviewed by McGinnis et al., 1990a) is one of the best characterized molecularly and developmentally. It is expressed in parasegments 0,1 and part of the 2 and plays a critical role in determining the identity of the maxillary segment. $D f d$ is a highly conserved gene; homologs of the Drosophila $D f d$ have been detected in a variety of species, including humans (Boncinelli et al., 1988; Duboule and Dollé, 1989). Moreover, one of the human homologs, Hox-4.2, if introduced in Drosophila, exerts a regulatory role similar to that performed by the endogenous Drosophila Dfd gene (McGinnis et al., 1990b), suggesting a evolutionary conservation of the $D f d$ function.

The interactions of $U b x$ with $D f d$ appear to be the exception to the rule of the phenotypic suppression by the more posterior acting gene; overexpression of a heat-shockdriven Ubx product does not completely suppress the endogenous $D f d$ gene (González-Reyes and Morata, 1990), even though $U b x$ acts much more posteriorly than $D f d$. Moreover, heat-shock experiments in which the Dfd protein is made ubiquitously also point to the same conclusion; structures like cirri, pattern elements characteristically determined by $D f d$ (Kuziora and McGinnis, 1988a), appear in the labial and the three thoracic segments of heat-shocked larvae, in spite of the fact that these segments contain Scr, Antp and Ubx proteins (see Akam, 1987 for review). However, in the abdominal segments, where other homeotic genes are active, the presence of ectopic Dfd protein does not result in differentiation of cirri, suggesting that some combinations of homeotic genes can suppress $D f d$. Since the rule of the phenotypic dominance of the posterior genes applies to evolutionary distant species (Duboule, 1991; Lufkin et al., 1991), apparent exceptions to this rule are worth investigating, for they may reveal insights into the phenomenon. By using a $h s p 70-D f d$ gene (Kuziora and McGinnis, 1988a), the $D f d$ gene can be coexpressed with other homeotic products. Although these interactions do not occur in vivo, the conditions in which $D f d$ is expressed or suppressed may help to understand how the homeotic genes operate in normal development.

$D f d$ also offers another aspect of particular interest, for it has been shown (Kuziora and McGinnis, 1988a) that heatshock-induced Dfd protein can trigger the expression of the $D f d$ endogenous gene in body regions where it is not normally activated. This out-of-context activation must be dif- ferent from the initial activating machinery and may reflect a secondary tier of regulation that operates in the maintenance of $D f d$ expression in the normal domain.

We have studied the phenomenon of autoactivation of $D f d$ and its dependence on polarity and homeotic genes, and also the interactions of the Dfd product with other homeoproducts. Our results indicate that the autoactivation of $D f d$ requires the functions of the genes wingless ( $w g$ ) and engrailed (en), and is inhibited by several homeoproducts. We also show that $D f d$ is phenotypically suppressed by high levels of $S c r, A n t p$ and $U b x$, and by the two forms of $A b d-B$ proteins.

\section{Material and methods}

\section{Mutant stocks and crosses}

The $h s p 70-D f d$ gene (HSD) was obtained from Drs Kuziora and McGinnis. The hsp70-Antp (HSA) and the hsp70-Ubx lines have already been described (Gibson and Gehring, 1988; GonzalezReyes et al., 1990). The $h s p 70-a b d-A$ stock has recently been made in our laboratory (Sampedro et al., unpublished data). A number of stocks were made that incorporate the HSD gene in different mutant combinations for polarity and homeotic genes. Mutations at polarity genes $e n, w g, p t c$ and $n k d$ were: $D f(2 R) e n^{B}$ (Gubb, 1985), wgCX4 (Baker, 1987), ptc ${ }^{I N}$ (Nüsslein-Volhard et al., 1984) and $n k d^{7 E 89}$ (Jürgens et al., 1984). As ANT-C mutations we used $\mathrm{Scr}^{\mathrm{Cl}}$ (Struhl, 1982) and $\mathrm{lab}^{f 40}$ (Merrill et al., 1989), which is mutant for $l a b$ and Antp. For the BX-C individual mutations, $U b x^{l}$ (Lewis, 1978), $a b d-A^{M 1}$ (Sánchez-Herrero et al., 1985), $A b d-B^{M 1}$ and $A b d-B^{M 5}$ (Casanova et al., 1986). In addition, the multiple mutant combinations used were: $S_{c r}{ }^{C 1} A n t p^{N S+R C 3}$ (Struhl, 1982),

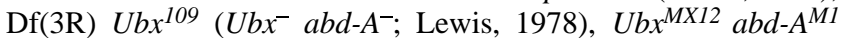
$A b d-B^{M 8}\left(U b x^{-} a b d-A^{-} A b d-B\left(m^{-} r^{-}\right)\right)$and $U b x^{M X 16} a b d-A^{M 1} A b d-$ $B^{M 9}$ (Ubx- abd-A- $A b d-B\left(m^{-} r^{+}\right)$; Casanova et al., 1987). The combinations $A n t p^{-}$and $U b x^{-} a b d-A^{-} A b d-B\left(m^{-} r^{+}\right)$were obtained after crossing $l a b^{f 40} / T M 3 \times S^{2} r^{C l} A n t p^{N S+R C 3} / T M 3$ and $U b x^{M X 12} a b d-A^{M 1} A b d-B^{M 8} / T M 6 B \times U b x^{M X 16} a b d-A^{M 1} A b d-$ $B^{M 9} / T M 6 B$, respectively.

\section{Heat-shock treatments}

The general rule was a single pulse of 1 hour at $36^{\circ} \mathrm{C}$ administered to embryos of 3 hours of age, following the protocol of Kuziora and McGinnis (1988a). In the case of the double heat shock with HSD and HSU or HSA, the embryos were given two 1 hour pulses to ensure a strong effect of the HSU and HSA genes (González-Reyes and Morata, 1990; unpublished observations).

\section{Antibody staining}

The protocol that we have used is described in Macías et al. (1990). For double labelling, we followed the protocol reported in Lawrence et al. (1987). The embryos, after dehydration, were embedded in Araldite (Fluka)/acetone (1:1) and dissected under the microscope. Then, mounted in Araldite. The polyclonal $D f d$ antibody was provided by Dr Paul Macdonald.

\section{Cuticular preparations of embryos}

First instar larvae were dechorionated in commercial bleach and the vitelline membrane removed using heptane:methanol (1:1). Then, after washing with methanol and $0.1 \%$ Triton $\mathrm{X}-100$, the larvae were mounted in Hoyer's:lactic acid (1:1) and allowed to clear at $60^{\circ} \mathrm{C}$ for at least 2 hours. The cuticles were observed under phase-contrast optics. 


\section{Results}

Ectopic activation of the endogenous Dfd gene by the Dfd product in the presence of a normal complement of homeotic genes

As shown by Kuziora and McGinnis (1988a), heat induction of the Dfd product driven by the HSD gene first results in a generalized presence of Dfd protein, but after 3 hours it is restricted to groups of ventral cells in the thoracic and abdominal segments. This, in addition to the normal $D f d$ domain (parasegments 0 and 1 and part of 2, McGinnis et al., 1990a). These authors also showed that the expression in the thorax and abdomen is due to inappropriate activation of the endogenous $D f d$ gene. They reported, and we have confirmed, that heat shocks given after 3 hours of development fail to produce morphological effects, suggesting the requirement of some additional factor(s) present in 3 hour embryos but not later.

We have investigated the compartmental origin of the cells showing ectopic activation of $D f d$ by double labelling with anti-en and anti-Dfd antibodies. A typical case is shown in Fig. 1A; the cells containing $D f d$ protein are immediately anterior to those expressing en with little or no overlap between them. The only exception to this rule occurs in the intersection of parasegments 2 and 3 (in the labial segment. Fig. 2A) where there are some cells coexpressing en and $D f d$. Thus, the ectopic but localized expression of $D f d$ appears in the anterior compartments and coincides in part with that of wingless (Van den Heuvel et al., 1989) at the extended germ band period.

The Dfd protein is present in stripes of cells of the ventral ectoderm. The pattern of expression is similar but not

Fig. 1. Location of $D f d$-expressing cells and ectopic cirri after heat shock. (A) The picture shows three abdominal metameres at the extended germ band stage after double staining for en (brown) and $D f d$ (blue). There is little or no overlap for the two labels, indicating that $D f d$ is activated in anterior compartment cells predominantly or exclusively. Note that the $D f d$ label is approximately coincident with $w g$ expression. The parasegmental boundary of one metamere is shown (A, anterior; $\mathrm{P}$, posterior).

(B) Position of the heat-shock-induced cirri (arrowheads) relative to that of the Keilin's organs (arrows), which mark the position of the anteroposterior compartment (parasegment) boundary in the embryonic ventral cuticle. In the thoracic and abdominal segments, the cirri always appear in the same position, in the middle of the naked region, very close to the parasegmental border.

Fig. 2. Effect of polarity genes on $D f d$ activation. (A, B) HSD embryo, otherwise normal, doubly stained for $D f d$ (ochre) and en (blue) proteins, showing the bands of ectopic $D f d$ activity. (C) HSD $w g^{-}$embryo stained as in (A); the ectopic $D f d$ expression is suppressed, and en is reduced. (D, E) HSD/HSW embryo

exhibiting a substantial increase in $D f d$ activity that in some cases covers most of the segment, including the posterior compartments. Staining as in (A). (B, E) Magnifications of the posterior ends of the embryos in (A) and (D) to show that the gain in $D f d$ expression in HSD/HSW is restricted to parasegment 13. No Dfd protein can be seen at or posterior to the 14th en stripe $(\mathrm{A} 8 \mathrm{p})$. identical in different parasegments (Fig. 3C,D). In parasegments 2 and 3 , numerous cells express $D f d$, whereas parasegment 4 shows much less expression. From 5 to 12 the amount of $D f d$ expression is approximately the same, it is reduced in 13 and absent in 14 and the rest of the body.

The morphological effects of the ectopic function of $D f d$ have been described by Kuziora and McGinnis (1988a); the three thoracic segments show cirri and sometimes mouth hooks, pattern elements characteristic of the maxillary segment (Jürgens et al., 1986; González-Reyes and Morata, 1991). The cirri always appear in the same region of the segment, approximately in the middle of the naked zone posterior to the denticle belt. From their position relative to that of the Keilin's organs (Fig. 1B), which mark the position of the anteroposterior compartment (parasegmental) boundary (Struhl, 1984), it can be seen that they originate from a region close to the border. This is approximately the same region where, as we showed above, $D f d$ becomes ectopically activated, indicating that the cirri originate from cells of, or close to, the $D f d$-expressing region. In some cases, however, they differentiate just posterior to the Keilin's organs (Fig. 1B), suggesting a posterior origin.

Previous results (Jürgens et al., 1986; González-Reyes and Morata, 1990) indicate that cirri and mouth hooks derive from the maxillary segment and, within it, from the posterior compartment (González-Reyes and Morata, 1991); in pair-rule mutants even-skipped (eve), that lack parasegment $1\left(\mathrm{Md}_{\mathrm{p}}-\mathrm{Mx}_{\mathrm{a}}\right)$, cirri and mouth hooks develop, whereas in fushi tarazu ( ftz) mutants lacking parasegment $2\left(\mathrm{Mx}_{\mathrm{p}}-\mathrm{Li}_{\mathrm{a}}\right)$, these structures are missing. In the latter experiments, the mutant embryos also contained the hsp70-Ubx gene so that after heat shock the head fails to involute, thus facilitating the identification and segmental allocation of head structures (González-Reyes and Morata, 1991). The observation that cirri originate in posterior compartments is an intriguing one for, as we show above, the ectopic expression of $D f d$ is restricted to anterior cells.

It is also of interest to note the lack of morphological effects in the abdominal segments, even though the amount of $D f d$ activity is similar in the thoracic and abdominal segments (Fig. 3C,D).

\section{The role of polarity and homeotic genes in the ectopic activation of Dfd}

The reason for studying the effects of these genes was that the $D f d$ ectopic expression shows a segmental periodic pattern, and differences attributable to segment identity, suggesting that both the polarity and homeotic genes may influence the activation of $D f d$.

We have studied the effects of variations in the expression of four major polarity genes, namely, wingless $(w g)$, engrailed (en), patched (ptc) and naked (nkd). Due to the difficulty of studying larval morphology in these mutant embryos, we have only considered the effects of these mutations on the expression of $D f d$ at the elongated germ band stage as visualized by the anti- $D f d$ antibody.

We first considered the effects of alterations in $w g$ function. In the $w g^{C X 4}$ mutant, a null allele (Baker, 1987), the normal domain of $D f d$ expression in the head is largely unaffected, although it becomes narrower than in wild-type 
embryos. However, the ectopic bands of $D f d$ expression fail to appear after heat shock, although a few cells in the labial segment express Dfd protein (Fig. 2C). This result is consistent with the position of the bands of $D f d$-expressing cells in part of the wingless domain and suggests a role of $w g$ in the autoactivation of $D f d$. This suggestion is strongly supported by the following experiment in which, taking advantage of a heat-shock $w g$ gene (HSW; Noordermeer et al., unpublished data), both $w g$ and $D f d$ were overexpressed at the same time. In this experiment, we observe an increase

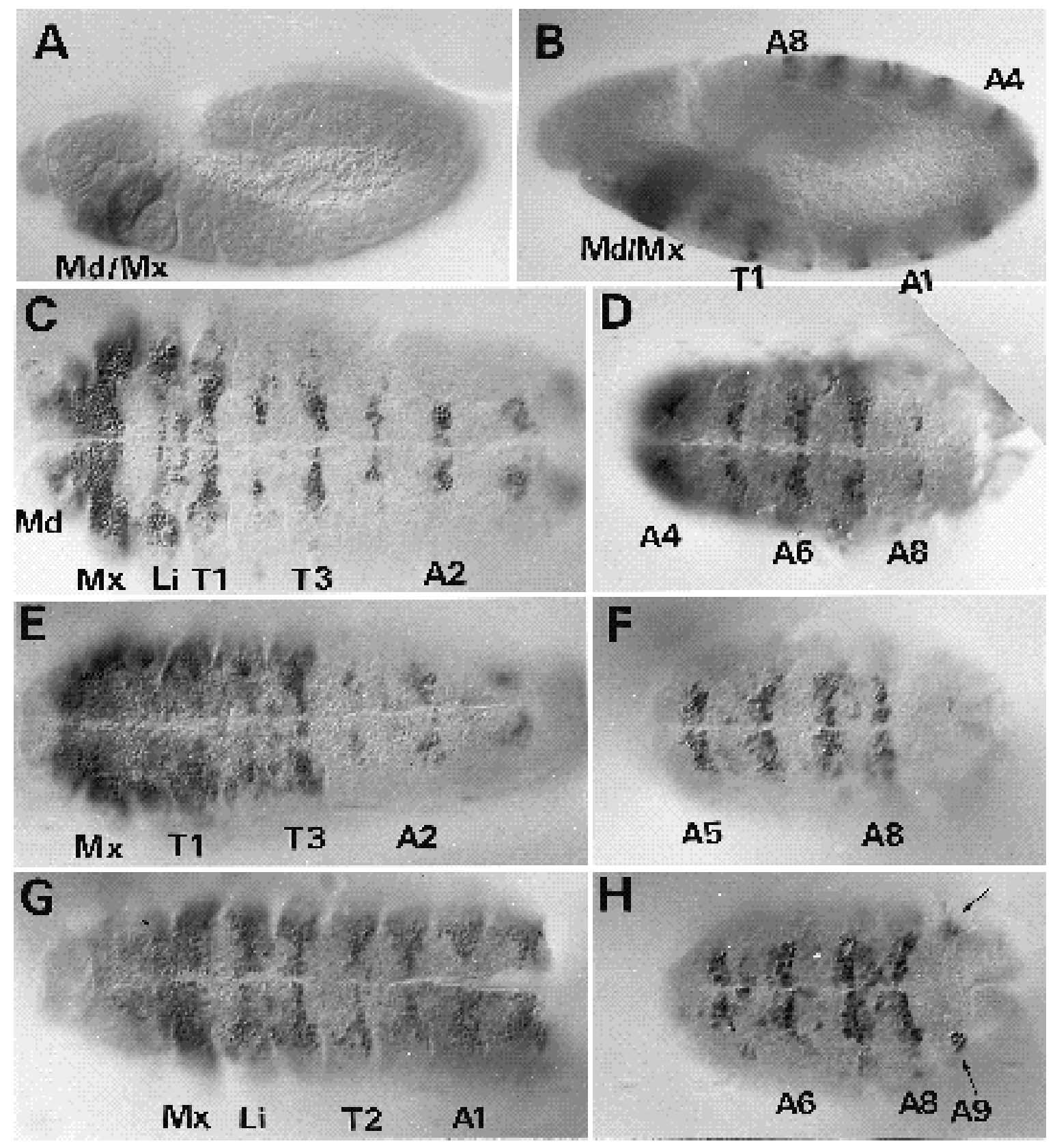

Fig. 3. Ectopic activation of $D f d$ after heat shock in embryos with a normal dose of the BX-C and in several mutant combinations. (A) Lateral view of the normal expression of $D f d$ in an HSD embryo at the extended germ band stage in the absence of heat shock; the protein is mainly restricted to mandibular and maxillary segments. (B) View of an HSD embryo of the same age after heat shock to show the periodic pattern of $D f d$ ectopic activation. (C, D) Ventral views of different regions of embryos like that of (B) to show more clearly the extent of $D f d$ expression. Note that the amount of protein in the labial (Li) and T1 segments is greater than in the other thoracic and abdominal segments, and also that there is very little expression in A8 and none in A9. (E) $D f d$ ectopic expression in $S c r^{-} A n t p^{-}$embryos. $^{-}$ The increase of $D f d$ protein is restricted to the T1 to T3 region. (F) $D f d$ expression in an $A b d-B\left(m^{-} r^{+}\right)$embryo. The number of cells expressing the protein is increased in parasegment 13 but not in 14. (G) Anterior region of an embryo deficient for $S c r$, Antp and the three BX-C genes. All the thoracic and abdominal segments exhibit the same and much increased activity of Dfd. (H) $D f d$ expression in the posterior region of an $A b d-B\left(m^{-} r^{-}\right)$embryo. The amount of $D f d$ product in parasegment 13 is greater than in the presence of the normal gene, and also extends to parasegment 14 (arrows). 
in the number of $D f d$-expressing cells outside the normal $D f d$ domain (Fig. 2D, E), pointing to a direct role of $w g$ in the process. However, not all the cells show $D f d$ expression indicating the existence of other factors involved in $D f d$ activation. In particular, there are no $D f d$-expressing cells at, or posterior to, the 14th en stripe (Fig. 2E).

In the case of engrailed, the complete lack of normal function in $D f(2 R) e n^{B}$ (Gubb, 1985) also results in lack of ectopic $D f d$ expression, even though it normally occurs in the anterior compartments. The domain of $D f d$ expression in $e n^{-}$embryos has been described (Jack et al., 1988): in comparison with the wild type, the lateral maxillary cells fail to express $D f d$, while the rest appears normal. In contrast with the $w g$ experiment, the co-overexpression of en and $D f d$ (using the heat-shock en (HSE) gene; Poole and Kornberg, 1988) does not result in an enhancement in $D f d$ activation, suggesting that the effect of en is indirect, probably through its effect on $w g$ (Martinez-Arias et al., 1988). In the other combinations involving patched and naked mutations, we do not find much difference with the standard situation containing the wild-type alleles of these genes. In HSD $p t c^{-}$we frequently observe that the area of ectopic $D f d$ expression fills the zone between normal en stripe and the ectopic en band anterior to it (Martinez-Arias et al., 1988), that is, the region of expanded $w g$ activity.

The role of homeotic genes on the autoactivation of $D f d$ was studied by heat inducing the HSD gene in various mutant combinations for several homeotic genes: Scr, Antp, $U b x, a b d-A$ and $A b d-B$.

We first studied the pattern of $D f d$ activation in embryos lacking Scr, Antp and the three BX-C genes. The results are illustrated in Fig. 3G; in comparison with embryos containing a full set of homeotic genes, the number of cells expressing $D f d$ is greatly increased. Also, the ectopic activation of $D f d$ now extends to parasegment 14 . Not all the cells of these metameres show detectable presence of $D f d$ antigen, suggesting that there are additional factors inhibiting $D f d$ activation. The number of $D f d$-expressing cells in the thoracic and A1 to A8 segments is approximately the same. These results demonstrate a repressing role of the ANT-C and BX-C genes on heat-induced ectopic $D f d$ expression.

Different homeotic genes have specific local effects on $D f d$ expression. For example, embryos lacking $S c r$ and Antp activities show high levels of ectopic $D f d$ expression in parasegments 4 and 5 (Fig. 3E), but the expression in the abdomen is like that of embryos containing a normal dose of these genes. The effect of individual Scr and Antp mutations is restricted to the domains of those genes. In the absence of the $U b x$ and $a b d-A$ genes, there is an increased level of product in the abdominal parasegments, except in 13 . Note the effect of $A b d-B$ mutations in the more posterior segments. In $A b d-B^{M 5}$, a $m^{-} r^{+}$mutation (Casanova et al., 1986), the ectopic expression of $D f d$ is still limited by the posterior border of parasegment 13, but the level of expression is higher than in the presence of the normal $A b d-$ $B$ gene (Fig. 3F). In $A b d-B^{M 1}$, a $m^{-} r^{-}$allele, the ectopic expression of $D f d$ in parasegment 13 is the same as in the previous case, but it now extends to parasegment 14 (Fig. $3 \mathrm{H})$.

\section{Developmental interactions of Dfd with other homeoproducts; conditions under which Dfd is phenotypically expressed or suppressed}

We next analyzed the phenotypic expression of the $D f d$ gene in cells containing various homeoproducts at different levels. Cirri and sometimes mouth hooks are used as morphological markers of $D f d$ expression. We utilized a segmental rather than a parasegmental description because cirri originate from just the parasegmental border and cells at both sides of it appear to be involved in their differentiation (see below).

In embryos deficient for Scr and Antp, cirri and mouth hooks are more numerous, in the prothoracic and mesothoracic segments, than in embryos containing these two genes (Fig. 4B). There is sometimes a piece of sclerotic tissue accompanying the cirri. These results clearly indicate that the wild-type $S c r$ and Antp genes partially suppress $D f d$ ectopic expression in the pro and mesothoracic segments. In $A n t p^{-}$larvae there is also an increase in $D f d$ phenotypic expression in the prothoracic and mesothoracic segments, but the effect in the prothorax is not as strong as in the absence of Scr.

The effects of the BX-C genes on the phenotypic expression of $D f d$ are schematized in Table 1. In the complete absence of BX-C function (row 1 in Table 1), Dfd causes the appearance of cirri in all thoracic and abdominal segments (Fig. 4C,G), including a set in the A9 segment.

The sole presence of the $r$ function (row 2) eliminates cirri in A9 (Fig. 4H), indicating that the $\mathrm{r}$ product alone is able to suppress $D f d$ expression, as expected from previous studies (Casanova et al., 1986; González-Reyes and Morata, 1990). The presence of a full dose of the $A b d-B$ gene, con-

Table 1. Presence (+) or absence (-) of cirri in thoracic (T) and abdominal (A) segments after heat induction of the HSD gene in different combinations for $B X-C$ genes

\begin{tabular}{|c|c|c|c|c|c|c|c|c|c|c|c|c|}
\hline Genotype & $\mathrm{T} 1$ & $\mathrm{~T} 2$ & $\mathrm{~T} 3$ & A1 & A2 & A3 & A4 & A5 & A6 & A7 & A8 & A9 \\
\hline (1) $\mathrm{Ubx}^{-} \mathrm{abd}-\mathrm{A}^{-} \mathrm{m}^{-} \mathrm{r}^{-}$ & + & + & + & + & + & + & + & + & + & + & + & + \\
\hline (2) $\mathrm{Ubx}^{-}$abd-A- $\left.\mathrm{A}^{-}\right\urcorner^{+}$ & + & + & + & + & + & + & + & + & + & + & + & - \\
\hline (3) $\mathrm{Ubx}^{-}$abd-A- $\mathrm{A}^{-} \mathrm{m}^{+}$ & + & + & + & + & + & + & + & + & + & + & - & - \\
\hline (4) $\mathrm{Ubx}^{-} \mathrm{abd}-\mathrm{A}^{+} \mathrm{m}^{+} \mathrm{r}^{+}$ & + & + & + & + & + & + & + & + & + & + & - & - \\
\hline (5) $\mathrm{Ubx}^{+}$abd-A- $\mathrm{m}^{+} \mathrm{r}^{+}$ & + & + & + & - & - & - & - & - & - & - & - & - \\
\hline (6) $\mathrm{Ubx}^{+}$abd-A $\mathrm{A}^{+} \mathrm{m}^{-} \mathrm{r}^{-}$ & + & + & + & - & - & - & - & - & - & - & + & + \\
\hline (7) $\mathrm{Ubx}^{+}$abd-A $\mathrm{A}^{+} \mathrm{m}^{-} \mathrm{r}^{+}$ & + & + & + & - & - & - & - & - & - & - & + & - \\
\hline (8) $\mathrm{Ubx}^{+}$abd-A $\mathrm{A}^{+} \mathrm{m}^{+} \mathrm{r}^{+}$ & + & + & + & - & - & - & - & - & - & - & - & - \\
\hline
\end{tabular}




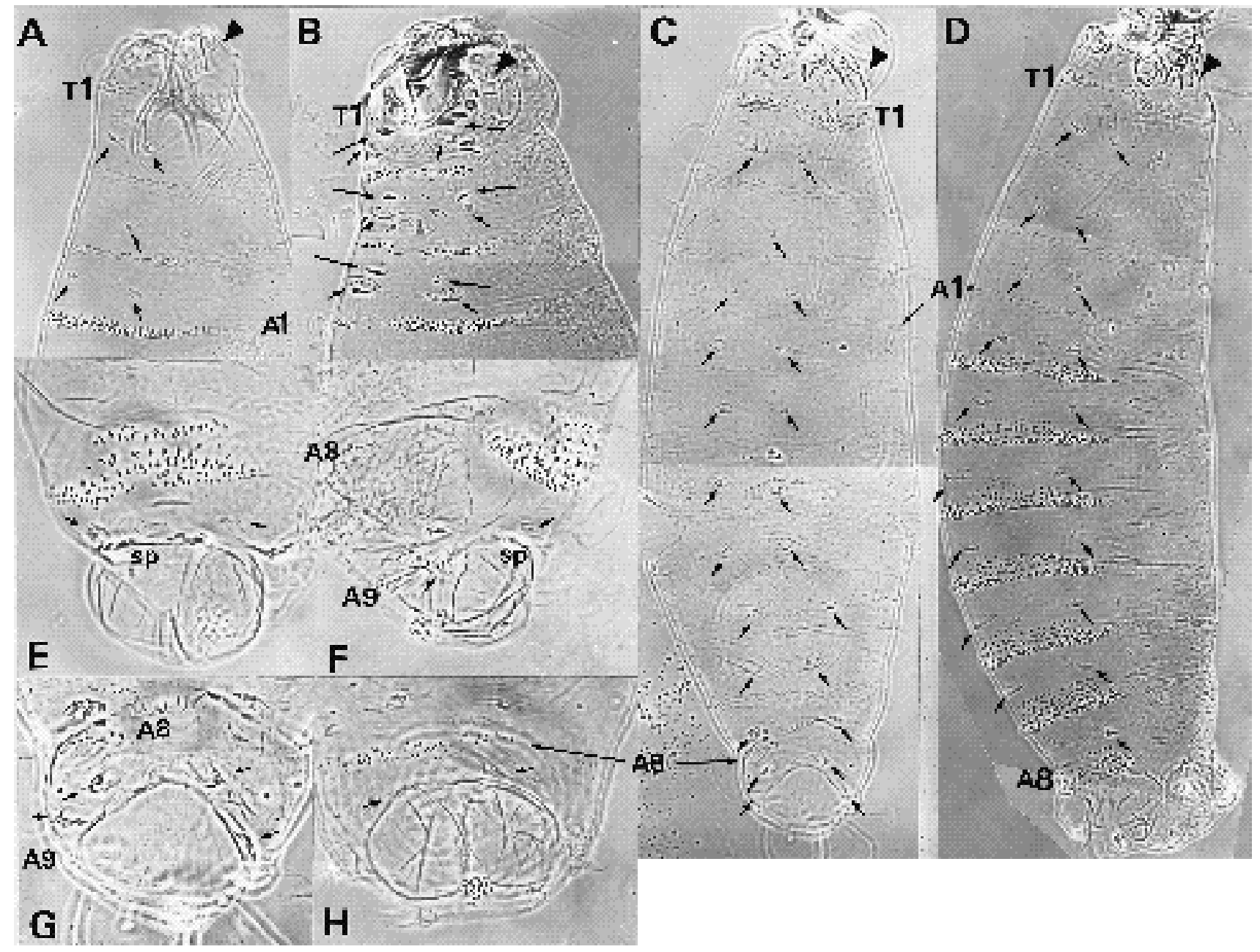

Fig. 4. Phenotypic expression of $D f d$ in several mutant combinations for homeotic genes. (A) Morphological effect of the HSD gene in a larva with a normal dose of homeotic genes. Cirri (arrows) appear in the middle of the naked region inbetween the ventral denticle belts. (B) HSD Scr-Antp- larva. Note the greater morphological effects; there are cirri (small arrows) and pieces of mouth hooks (big arrows). (C) HSD BX-C ${ }^{-}$larva. Cirri (arrows) are present in all abdominal segments including an extra set in A9. (D) HSD Ubx larva. Cirri (arrows) appear in the thorax and in the A1 to A7 segments. (E, F) Ventral and lateral view of a HSD $A b d-B\left(m^{-} r^{-}\right)$larva. The cirri, indicated by the arrows in E, belong to the A8 segment, whereas those of A9 are in a more lateral position, as indicated by the left arrow in F. (G) Higher magnification of the posterior end of the larva in C to show the two sets of cirri (arrows) of A8 and A9. To be compared with H. HSD BX-C $-\mathrm{r}^{+}$larva showing cirri (arrows) in A8 but absence in A9. In all cases, arrowheads mark the normal position of the cirri in the maxillary segment

taining the $m$ and $r$ functions (row 3), suppresses cirri both in A8 and A9 (Fig. 4D). Since $m$ function is limited by the posterior border of parasegment 13 (A7p-A8a), and it is in this parasegment where it has the highest level of expression (Kuziora and McGinnis, 1988b; Sánchez-Herrero and Crosby, 1988; Celniker et al., 1989; DeLorenzi and Bienz, 1990), this indicates that it is the high levels of $\mathrm{m}$ product in A8a that suppress $D f d$. In contrast, lower levels of $\mathrm{m}$ product in the A5-A7 abdominal region (Kuziora and McGinnis, 1988b; Sánchez-Herrero and Crosby, 1988; Celniker et al., 1989; DeLorenzi and Bienz, 1990) cannot achieve it (Fig. 4D). In the complementary combination (row 6), lacking the $m$ and $r$ functions, cirri appear in A8 and A9, but not in the other abdominal segments (Fig. 4E,F). The specificity of the $m$ function for the appearance of cirri in A8 was further tested in another com- bination (row 7), which possesses all BX-C functions but that of $m$. In these larvae cirri develop in A8.

The roles of $a b d-A$ and $U b x$ were tested in other combinations (Table 1, rows 4, 5 and 8). The phenotypes of these embryos clearly indicate that the presence of cirri is independent of $a b d-A$, but strongly dependent on $U b x$ (Fig. 4D).

Co-overexpression of $\mathrm{Dfd}$ with Antp, with $\mathrm{Ubx}$, and with abd-A

The previous results indicate that cirri can appear in segments T2 and T3 which contain Antp and Ubx products, although the expression of $U b x$ in T3 is characteristically low (White and Wilcox, 1985). We have also found that, in the absence of $U b x, a b d-A$ is not able to suppress cirri in the A1-A7 segments. In principle, this is against the expectations because, following the rule of the suppression 
by the more posterior gene, these genes should suppress $D f d$. We have tested whether the lack of suppression may be due to an insufficient amount of product in the place where $D f d$ becomes activated. Using the respective (HSA, HSU and $h s p 70-a b d-A$, termed HSAA) heat-shock genes, we can add together the endogenous and the heat-shockdriven product for these genes and study how they affect the differentiation and disposition of cirri.

In the HSD/HSA experiment, we observe a transformation of the cephalic and the T1 segment toward T2, as expected for generalized expression of the Antp product (Gibson and Gehring, 1988; González-Reyes et al., 1990). However, after having examined several hundred embryos, there were only two cases showing cirri, one in T1 and the other in T2, indicating a suppressing role of the Antp product when present in high amounts.

For $a b d-A$ (HSD/HSAA experiment), we observe a similar result; the high, uniform level of $a b d-A$ induced by the heat shock results in the suppression of cirri in the thorax: only five out of 118 embryos showing abdominal (HSAAinduced) transformation present cirri in the thorax. In contrast, about $80 \%$ of the HSD/+ embryos present cirri. This result is apparently paradoxical because the endogenous $a b d-A$ gene is unable (Table 1) to suppress $D f d$ in the abdominal segments. One possible reason is that the level of the endogenous $a b d-A$ is very low in the region anterior to the parasegmental boundary, just where $D f d$ is ectopically activated. To test this possibility, we have heatinduced $a b d-A$ and $D f d$ in the absence of the endogenous $U b x$ and $a b d-A$ genes. The result is that the high and uniform level of the heat-induced abd-A product is able to suppress $D f d$ : of a total of $25 \mathrm{HSD} / \mathrm{HSAA} U b x^{-} a b d-A^{-}$ embryos, only seven show some cirri, and these all were embryos showing mild abdominal transformation, that is, those in which the HSAA gene had not been highly expressed. Interestingly, the great majority of cirri appeared in the abdominal segments.

However, in the case of $U b x$ (HSD/HSU experiment), we frequently find that the heat-shocked embryos show the sum of the $D f d$ and $U b x$ induced transformations. For example, $13 / 52(25 \%)$ of the HSD/HSU larvae showed cirri, in the thoracic segments transformed into A1. Although this frequency of transformation is lower than in the HSD/+ genotype, which is around $80 \%$, we often observe cirri even in cases of extreme A1 transformation, which require high levels of Ubx product.

\section{Discussion}

\section{Ectopic autoactivation of $\mathrm{Dfd}$}

All the developmental effects of the HSD gene are mediated by the endogenous $D f d$ gene (Kuziora and McGinnis, 1988a), which becomes inappropriately activated by the exogenous Dfd product. Since the normal activating process (Jack et al., 1988; Jack and McGinnis, 1990) cannot require the presence of Dfd product, this must be a different procedure to trigger $D f d$ expression, which has its own specific activating machinery. There are three factors involved in the process: (1) the $D f d$ protein, (2) some polarity genes, especially $w g$, and (3) the products of several homeotic genes of the ANT-C and BX-C. Since these alone do not account for all the facts observed, there must be other elements involved in the process.

The role of the Dfd protein is well established by McGinnis' group (see review by McGinnis et al., 1990a), the autocatalytic sites have been found in the $D f d$ promoter. We deal here with the roles of polarity and homeotic genes in the process.

Our results indicate that $w g$ plays an important role in the ectopic expression of $D f d$, which is suppressed in $w g$ mutants and enhanced after heat induction of the HSW gene (Fig. 2). In particular, the heat-shock experiment is significant for it shows that just the Dfd and wg products alone are able to trigger the mechanism of $D f d$ autoactivation. wg appears to be required only in the early phase of the activation process, because the heat-shock-driven wg product lasts only about 1 hour (Noordermeer et al., unpublished data) and the endogenous $D f d$ gene remains active for several hours after the heat shock. In this context, it is of interest to note that $w g$ is also involved in the autocatalysis of $U b x$ in the visceral mesoderm (Thuringer and Bienz, personal communication) and plays a role in the activation of labial in the visceral mesoderm (Immergluck et al., 1990). The lack of ectopic $D f d$ expression in en mutants may be explained by the loss of $w g$ function in these mutants (DiNardo et al., 1988; Martinez-Arias et al., 1988). This interpretation is consistent with the lack of effect of $e n$ overexpression on $D f d$ autoactivation. We have not studied the effect of other polarity genes (apart from ptc and $n k d$ ), but the fact that the ectopic $D f d$ expression is not exactly coincident with $w g$ expression, suggests that others of these genes might be involved. The temporal specificity (3 hours of development) of the induction of $D f d$ autocatalysis also indicates the requirement of some early factor that cannot be $w g$, present through all the embryonic period (Van den Heuvel et al., 1989). Altogether, these observations may indicate a possible role for polarity genes in the control of homeotic function after their initial activation. This view is also consistent with the temporal pattern of activity of many polarity genes, which spans embryonic and larval life, just like the homeotic genes (see review by Wilkins and Gubb, 1991).

$D f d$ autoactivation has been studied in various mutant combinations for different homeotic genes. In the absence of Scr, Antp and the BX-C genes, the amount of $D f d$ activity and the extent of its morphological effect is greatly increased (Fig. 3G), indicating that their wild-type alleles have a repressing role. Their specific effect is local and according to their particular pattern of expression. The elimination of $S c r$ and Antp raises $D f d$ expression in T1 and T2 segments, but there is no alteration in the abdominal segments. Conversely, the elimination of the BX-C genes produces an increase limited to the abdominal segments. The individual contribution of each homeotic gene to $D f d$ repression is variable; the wild-type allele of $U b x$ reduces but does not completely eliminate $D f d$ activity, which spreads along the $U b x$ domain (Fig. 3). Similarly, the $a b d$ $A$ gene has little effect on the ectopic activation of $D f d$, possibly because the part of the segment where $D f d$ is more 
strongly expressed contains low expression of $a b d-A$ (Karch et al., 1990; Macías et al., 1990).

The $A b d-B$ gene has a clearer effect on $D f d$ activity. The $m$ element reduces it to a very low level in parasegment 13, and the $r$ element eliminates it completely in parasegment 14 (Fig. 3D). It is significant that the enhancing effect of the HSW gene is restricted to the $m$ domain, but cannot transgress the repressing effect of the $r$ element (Fig. 2), emphasizing once again the strong capacity of the $r$ product to repress any homeotic activity (Casanova et al., 1986; González-Reyes and Morata, 1990).

\section{Phenotypic suppression of Dfd by other homeotic genes: Dfd follows the rule of the phenotypic dominance by the more posterior gene - with some qualifications}

As stated in the Introduction, the expression of $D f d$ appears to be the exception to the rule of the phenotypic dominance by the posterior genes; when $D f d$ is expressed in thoracic segments that contain Scr, Antp and Ubx products, it can alter the identity of those segments, so that they differentiate structures like cirri, characteristic of a cephalic (maxillary) segment. The observation, however, that in the absence of $S c r$ and Antp the phenotypic expression of $D f d$ is increased, indicates that these two genes, at their normal level of expression, partially suppress $D f d$. Moreover, when Antp is expressed at higher levels, by using the heat-shock Antp gene, $D f d$ expression is completely eliminated.

The interactions of $D f d$ with $U b x$ also indicate a suppressing role of $U b x$. This gene is normally expressed from parasegment 5 to parasegment 13. The amount of product is very low in 5, high in 6 and then gradually decreases in posterior parasegments to a low level in 13 (White and Wilcox, 1985). Heat induction of $D f d$ in the presence of a normal $U b x$ gene, produces cirri in $\mathrm{T} 3$, but not in $\mathrm{A} 1$ or more posterior segments, suggesting that the high levels of $U b x$ in A1, but not the low ones in T3, suppress $D f d$. This is supported by the effect of HSD in $U b x^{-}$embryos, where cirri are found in all abdominal segments except in A8. As neither the endogenous $a b d-A$ nor $A b d-B$ prevent cirri differentiation in the A1 to A7 segments (see Table 1 and below), these experiments suggest that, just like Antp, $U b x$ alone is able to suppress $D f d$, provided that it is in a sufficiently high concentration.

However, to our surprise, high levels of $U b x$ product in the double heat-shock HSD/HSU only suppress cirri partially, even though a $U b x$ induced A1 transformation, requiring a high level of Ubx product, is observed. We do not have a satisfactory explanation for this; one possibility might be that, unlike the endogenous $U b x$ gene, the HSU only encodes a form of Ubx protein, which might be less efficient to suppress $D f d$ than the others. Besides, the exogenous Ubx protein only lasts for a few hours (González-Reyes and Morata, 1990), and this may not be sufficient to prevent the autocatalytic expression of $D f d$.

Of the homeotic genes studied, only the endogenous $a b d$ $A$ appears not to suppress $D f d$ expression. The presence of abd-A product in HSD $U b x^{-}$embryos does not prevent cirri differentiation in the $a b d-A$ expression domain. Our experiments strongly suggest that the reason for this lies in the differences of the expression domains of the heat-induced $D f d$ gene and the endogenous $a b d-A$. The ectopic expression of $D f d$ in HSD embryos is localized just anterior to the en stripe, precisely the place where the expression of $a b d-A$ is lowest (Karch et al., 1990; Macías et al., 1990), so the concentration of abd-A product is too low to suppress $D f d$. When this level is raised using the HSAA gene, then $D f d$ is suppressed. Moreover, the exogenous abd-A product can do it by itself as demonstrated by the HSD/HSAA $U b x^{-} a b d-A^{-}$experiment.

The phenotypic suppression of $D f d$ by the Abd-B products is very clear. The elimination of the $\mathrm{m}$ and $\mathrm{r}$ proteins in the null $A b d-B^{M I}$ mutant results in the development of cirri in A8 and A9, correlated with the increase or de novo presence of $D f d$ product in those segments. Using mutants defective in either $\mathrm{m}$ or $\mathrm{r}$ forms (Table 1), we can show that the appearance of cirri in A8 depends on the absence of the m protein, while in A9 it depends on $r$. It is worth noting that the $\mathrm{m}$ protein is also present in lower amounts in segments A5 to A7 (Celniker et al., 1989; DeLorenzi and Bienz, 1990), but it only suppresses $D f d$ in A8, indicating that the suppression of $D f d$ requires a high amount of $\mathrm{m}$ protein.

Thus, in all these cases, the correlation between pattern of expression and phenotypic suppression is maintained, but the repressing homeoproducts have to be in sufficiently high amounts. This is significant, for it suggests that the phenotypic suppression depends not only on the intrinsic property of the homeoproduct, but also on its concentration, which in turn favours a model of suppression based in competition for binding sites. One might speculate from these observations that one reason for the phenotypic suppression by the more posterior genes is simply that posteriorly acting homeoproducts are more concentrated in the cells.

\section{Transcriptional regulation versus phenotypic suppression between homeotic genes}

The results obtained with $D f d$ also bear on the problem of regulatory interactions between homeotic genes and the phenomenon of phenotypic suppression (González-Reyes and Morata, 1990). We find a correlation between transcriptional down-regulation and phenotypic suppression even though the latter can occur if transcriptional downregulation is impeded (González-Reyes et al., 1990). For example, in the case of HSD Scr-Antp ${ }^{-}$embryos, the increase in the morphological effect of the heat shock (Fig. 4B) correlates with an increased level of $D f d$ expression in parasegments 4 and 5 (Fig. 3E). Similarly, the presence of cirri in the abdominal segments in the different mutant combinations for the BX-C is accompanied by higher levels of $D f d$ protein in those segments (compare Figs 3, 4)

A possible explanation to link these phenomena is based on the mode of action of homeoproteins, which act as transcriptional activators by binding to the promoters of the genes they regulate: the downstream genes. This is the principal morphogenetic function of the homeotic genes and the one that ultimately establishes the identity of the different metameres. In addition, at least in some cases (Beachy et al., 1988; Müller et al., 1989; Regulski et al., 1991), the homeotic genes enhance and maintain their own transcription by autocatalysis; their products bind to their own promoters. 
We believe that both phenotypic suppression and transcriptional regulation may be the result of the same phenomenon of competition for binding sites. Phenotypic suppression may result if one of the homeoproteins has stronger affinity for the same binding sites of the downstream genes, thus dictating the identity. Transcriptional down-regulation may simply result from competition for transcriptional enhancing sites of the homeotic genes. The protein with strong affinity may block the enhancing function of the autocatalytic product. The case of the $\mathrm{r}$ protein of $\mathrm{Abd}-\mathrm{B}$ may illustrate the point. It may bind to the $U b x$ promoter more strongly than the Ubx protein, but may be unable to enhance $U b x$ transcription. In the case of the ectopic expression of $D f d$, the Antp or Abd-B proteins probably occupy the autocatalytic site and inhibit the maintenance circuit.

\section{Are cirri originated by induction across the parasegmental boundary?}

One striking aspect of the experiments reported in this paper concerns the origin of cirri. According to the following arguments, they should originate in the posterior compartment of the maxillary segment: (1) they often appear posterior to the Keilin's organ that marks the position of the parasegmental boundary (Struhl, 1984); (2) they are present in eve mutants, where parasegment $1\left(\mathrm{Md}_{\mathrm{p}}-\mathrm{Mx}_{\mathrm{a}}\right)$ is missing but the $\mathrm{Mx}_{\mathrm{p}}$ compartment is present; (3) they are lacking in $f t z$ mutants, where parasegment $2\left(\mathrm{Mx}_{\mathrm{p}}-\mathrm{Li}_{\mathrm{a}}\right)$ is missing.

However, the following arguments would suggest that their presence depends on the expression of $D f d$ in anterior compartment cells: (1) the appearance of cirri depends on the ectopic expression of the endogenous $D f d$ gene, which is, as we show above, restricted to the anterior compartments. (2) After heat induction of the HSD gene, the differentiation of cirri in A8 depends exclusively on the absence of the $m$ function (see Fig. 4 and Table 1), which ends at A8 a (Casanova et al., 1986; Boulet et al., 1991).

In our opinion, the best way to explain these observations is by invoking the process of induction, so that $D f d$ expressing cells in the anterior compartment induce the differentiation of cirri in cells immediately adjacent, but located at the other side of the parasegmental border. Interactions across parasegments are not unknown; $w g$ and en (DiNardo et al., 1988; Martinez-Arias et al., 1988) are mutually required to maintain their expression. Also, the differentiation of some embryonic and adult structures is based on an induction mechanism: the development of the gastric caeca requires $\mathrm{Scr}$ expression in the visceral mesoderm, although $\mathrm{Scr}$ is only present in cells posterior to the cells that form the gastric caeca (Reuter and Scott, 1990); the mother cell of the leg bristles induces a nearby cell to differentiate as a bract; cell marking shows that the induced cell originates from a different lineage (Garcia-Bellido, 1966). Similarly, the differentiation of the ommatidia in the eye is based on recruitment of cells of different lineages (Lawrence and Green, 1979).

Another possible explanation is that the cirri may originate from both anterior and posterior cells. This is also not without precedents; even a single pattern element like the 'bristle of doubt' in the antenna (Morata and Lawrence,
1979) can be differentiated by anterior or by posterior cells. Also, some bristles in the tarsi can have anterior or posterior provenance (Lawrence et al., 1979). We believe, however, that this explanation is unlikely here because we would have expected cirri to be present in both eve and $f t z$ mutants, and we find that they are lacking in $\mathrm{ftz}$.

We thank Isabel Guerrero, Mariann Bienz, Dolores MartínBermudo, Peter Lawrence, Bill McGinnis and Ernesto SánchezHerrero for their useful comments on the manuscript. We also thank Peter Lawrence for his gift of the hsp70-wg gene before publication. We also acknowledge the financial support of the Dirección General de Investigación Científica y Técnica and the institutional support from the Fundación Ramón Areces.

\section{References}

Akam, M. (1987). The molecular basis for metameric pattern in the Drosophila embryo. Development 101, 1-22.

Baker, N. (1987). Molecular cloning of sequences from wingless, a segment polarity gene in Drosophila: The spatial distribution of transcript in embryos. EMBO J. 6, 1765-1773.

Beachy, P., Krasnow, M., Gavis, E. and Hogness, D. (1988). An Ultrabithorax protein binds sequences near its own and the Antennapedia promoters. Cell 55, 1069-1081.

Bergson, C. and McGinnis, W. (1990). An autoregulatory enhancer element of the Drosophila homeotic gene Deformed. EMBO J. 9, 42874297.

Bienz, M. and Tremml, G. (1988). Domain of Ultrabithorax expression in Drosophila visceral mesoderm from autoregulation and exclusion. Nature 333, 576-578.

Boncinelli, E., Somma, R., Acampora, D., Pannese, M., D'Esposito, M., Faiella, A. and Simeone, A. (1988). Organization of human homeobox genes. HumanReproduction 3, 880-886.

Boulet, A., Lloyd, A. and Sakonju, S. (1991). Molecular definition of the morphogenetic and regulatory functions and the cis-regulatory elements of the DrosophilaAbd-B homeotic gene. Development 111, 393-405.

Busturia, A. and Morata, G. (1988). Ectopic expression of homeotic genes caused by the elimination of the Polycomb gene in Drosophila imaginal epidermis. Development 104, 713-720.

Carroll, S., Laymon, R., McCutcheon, M., Riley, P. and Scott, M. (1986). The localization and regulation of Antennapedia protein expression in Drosophila embryos. Cell 47, 113-122.

Casanova, J., Sánchez-Herrero, E., Busturia, A. and Morata, G. (1987). Double and triple mutant combinations of the bithorax complex of Drosophila. EMBO J. 6, 3103-3109.

Casanova, J., Sánchez-Herrero, E. and Morata, G. (1986). Identification and characterization of a parasegment specific regulatory element of the Abdominal-B gene of Drosophila. Cell 47, 627-636.

Celniker, S., Keelan, D. and Lewis, E. (1989). The molecular genetics of the bithorax complex of Drosophila: characterization of the products of the Abdominal-B domain. Genes Dev. 3, 1424-1436.

Chadwick, R. and McGinnis, W. (1987). Temporal and spatial distribution of transcripts from the Deformed gene of Drosophila. EMBO J. 6, 779789.

DeLorenzi, M. and Bienz, M. (1990). Expression of Abdominal-B homeoproteins in Drosophila embryos. Development 108, 323-329.

Desplan, C., Theis, J. and O'Farrell, P. (1985). The Drosophila developmental gene engrailed encodes a sequence-specific DNA binding activity. Nature 318, 630-635.

DiNardo, S., Sher, E., Heemskerk-Jongens, J., Kassis, J. and O'Farrell, P. (1988). Two-tiered regulation of spatially patterned engrailed gene expression during Drosophila embryogenesis. Nature 332, 604-609.

Dolle, P. and Duboule, D. (1989). Two genes of the murine HOX-5 complex show regional and cell-type specific expression in developing limbs and gonads. EMBO J. 8, 1507-1515.

Duboule, D. (1991). Patterning in the vertebrate limb. Curr. Opin. Genet. Dev. 1, 211-216.

Duboule, D. and Dollé, P. (1989) The structural and functional organization of the murine HOX gene family resembles that of Drosophila homeotic genes. EMBO J. 8, 1497-1505. 
Duncan, I. (1987). The bithorax complex. Ann. Rev. Genet. 21, 285-319.

García-Bellido, A. (1966). Pattern reconstruction by dissociated imaginal disk cells of Drosophila melanogaster. Dev. Biol. 14, 278-306.

Gehring, W. (1987). Homeoboxes in the study of development. Science 236, $1245-1252$.

Gibson, G. and Gehring, W. (1988). Head and thoracic transformations caused by ectopic expression of Antennapedia during Drosophila development. Development 102, 657-675.

González-Reyes, A. and Morata, G. (1990). The developmental effect of overexpressing a $U b x$ product in Drosophila embryos is dependent on its interactions with other homeotic products. Cell 61, 515-522.

González-Reyes, A. and Morata, G. (1991). Organization of the Drosophila head as revealed by the ectopic expression of the Ultrabithorax product. Development 113, 1459-1471.

González-Reyes, A., Urquia, N., Gehring, W., Struhl, G. and Morata, G. (1990). Are cross-regulatory interactions between homoeotic genes functionally significant? Nature 344, 78-80.

Gubb, D. (1985). Further studies on engrailed mutants in Drosophila melanogaster.Roux's Arch. Dev. Biol. 194, 236-246.

Hafen, E., Kuroiwa, A. and Gehring, W. (1984). Spatial distribution of transcripts from the segmentation gene fushi tarazu during Drosophila embryonic development. Cell 37, 833-841.

Immergluck, K., Lawrence, P. and Bienz, M. (1990). Induction across germ layers in Drosophila mediated by a genetic cascade. Cell 62, 261268

Jack, T. and McGinnis, W. (1990). Establishment of the Deformed expression stripe requires the combinatorial action of coordinate, gap and pair-rule proteins. EMBO J.9, 1187-1198.

Jack, T., Regulski, M. and McGinnis, W. (1988). Pair-rule segmentation genes regulate the expression of the homeotic selector gene, Deformed. Genes Dev. 2, 635-651.

Jürgens, G., Lehman, R., Schardin, M. and Nüsslein-Volhard, C. (1986). Segmental organization of the head and embryo of Drosophila melanogaster. Roux's Arch. Dev. Biol. 195, 359-377.

Jürgens, G., Wieschaus, E., Nüsslein-Volhard, C. and Kluding, $\mathbf{H}$ (1984). Mutations affecting the pattern of the larval cuticle in Drosophila melanogaster. Roux's Arch. Dev. Biol. 193, 283-295.

Karch, F., Bender, W. and Weiffenbach, B. (1990). abdA expression in Drosophila embryos. Genes Dev. 4, 1573-1587.

Krasnow, M., Saffman, E., Kornfeld, K. and Hogness, D. (1989). Transcriptional activation and repression by Ultrabithorax proteins in cultured Drosophila cells. Cell 57, 1031-1043.

Kuziora, M. and McGinnis, W. (1988a). Autoregulation of a Drosophila homeotic selector gene. Cell 55, 477-485.

Kuziora, M. and McGinnis, W. (1988b). Different transcripts of the Drosophila $A b d-B$ gene correlate with distinct genetic sub-functions. EMBO J. 7, 3233-3244.

Lawrence, P. A. and Green, S. (1979). Cell lineage in the developing retina of Drosophila. Dev. Biol. 71, 141-152.

Lawrence, P. A., Johnson, P., McDonald, P. and Struhl, G. (1987). Borders of parasegments in Drosophila embryos are delimited by the fushi tarazu and even-skippped genes. Nature 328, 440-442.

Lawrence, P. A., Struhl, G. and Morata, G. (1979). Bristle patterns and compartment boundaries in the tarsi of Drosophila. J. Embryol. exp. Morph. 51, 195-208.

Lewis, E. (1978). A gene complex controlling segmentation in Drosophila. Nature 276, 565-570.

Lewis, E. (1981). Developmental genetics of the bithorax complex in Drosophila. In Developmental Biology using Purified Genes, ICN-UCLA Symposia on Molecular and Cellular Biology (ed. F. Donald and F. Brown) Volume 23, pp.189-208. New York: Academic Press.

Lufkin, T., Dierich, A., LeMeur, M., Mark, M. and Chambon, P. (1991). Disruption of the Hox-1.6 homeobox gene results in defects in a region corresponding to its rostral domain of expression. Cell 66, 1105-1119.

Macías, A., Casanova, J. and Morata, G. (1990). Expression and regulation of the $a b d-A$ gene of Drosophila. Development 110, $1197-$ 1207.

Mahaffey, J. and Kaufman, T. (1988). The homeotic genes of the Antennapedia Complex and the bithorax Complex of Drosophila. In Developmental Genetics of Higher Organisms. A Primer in Developmental Biology (ed. G. M. Malacinski). Volume 14, pp. 329-360. New York: Macmillan Publishing Company.

Martínez-Arias, A. and Lawrence, P. A. (1985). Parasegments and compartments in the Drosophila embryo. Nature 313, 639-642.
Martinez-Arias, A., Baker, N. and Ingham, P. (1988). Role of segment polarity genes in the definition and maintenance of cell states in the Drosophila embryo. Development 103, 157-170.

McGinnis, W., Jack, T., Chadwick, R., Regulski, M., Bergson, C., McGinnis, N. and Kuziora, M. (1990a). Establishment and maintenance of position-specific expression of the Drosophila homeotic selector gene Deformed. In Advances in Genetics: Genetic Regulatory Hierarchies in Development (ed.T. R. F. Wright). Volume 27, pp. 363-402. San Diego: Academic Press.

McGinnis, N., Kuziora, M. and McGinnis, W . (1990b). Human Hox-4.2 and Drosophila Deformed encode similar regulatory specificities in Drosophila embryos and larvae. Cell 63, 969-976.

McGinnis, W. and Krumlauf, R. (1991). Homeobox genes and axial patterning. Cell 67, 283-302.

McGinnis, W., Levine, M., Hafen, E., Kuroiwa, A. and Gehring, W. (1984). A conserved DNA sequence in homeotic genes of the Drosophila Antennapedia and bithorax complexes. Nature 308, 428-433.

Merrill, V., Diederich, R., Turner, F. and Kaufman, T. (1989). A genetic and developmental analysis of mutations in labial, a gene necessary for proper head formation in Drosophilamelanogaster.Dev. Biol. 135, 376391.

Morata, G. and Lawrence, P. (1979). Development of the eye-antenna imaginal disc of Drosophila. Dev. Biol. 70, 355-371.

Müller, J., Thuringer, F., Biggin, M., Zust, B. and Bienz, M. (1989). Coordinate action of a proximal homeoprotein binding site and a distal sequence confers the Ultrabithorax expression pattern in the visceral mesoderm. EMBO J. 8, 4143-4151

Nüsslein-Volhard, C., Wieschaus, E. and Kluding, H. (1984). Mutations affecting the pattern of the larval cuticle in Drosophila melanogaster. Roux's Arch. Dev.Biol. 193, 267-282.

Poole, S. and Kornberg, T. (1988). Modifying expression of the engrailed gene of Drosophilamelanogaster.Development 104, 85-94.

Regulski, M., Dessain, S., McGinnis, N. and McGinnis, W. (1991). Highaffinity binding sites for the Deformed protein are required for the function of an autoregulatory enhancer of the Deformed gene. Genes Dev 5, 278-286.

Regulski, M., McGinnis, N., Chadwick, R. and McGinnis, W. (1987). Developmental and molecular analysis of Deformed; a homeotic gene controlling Drosophila head development. EMBO J. 6, 767-777.

Reuter, R. and Scott, M. (1990). Expression and function of the homeotic genes Antennapedia and Sex combs reduced in the embryonic midgut of Drosophila. Development 109, 289-303.

Sánchez-Herrero, E. and Crosby, M. (1988). The Abdominal-B gene of Drosophila melanogaster: overlapping transcripts exhibit two different spatial distributions. EMBO J. 7, 2163-2173.

Sánchez-Herrero, E., Vernos, I., Marco, R. and Morata, G. (1985). Genetic organization of the Drosophila bithorax complex. Nature 313, 108-113.

Scott, M. and Weiner, A. (1984). Structural relationships among genes that control development sequence homology between Antennapedia, Ultrabithorax and fushi tarazu loci of Drosophila. Proc. Natl. Acad. Sci. USA 81, 4115-4119.

Struhl, G. (1982). Genes controlling segmental specification in the Drosophila thorax. Proc. Natl. Acad. Sci.USA 79, 7380-7384.

Struhl, G. (1983). Role of esc product in ensuring the selective expression of segment-specific genes in Drosophila. J. Embryol. exp. Morph. 76, 297-331.

Struhl, G. (1984). Splitting the bithorax complex of Drosophila. Nature 308, 454-457.

Struhl, G. and White, R. (1985). Regulation of the Ultrabithorax gene of Drosophila by other bithorax complex genes. Cell 43, 507-519.

Van den Heuvel, M., Nusse, R., Johnston, P. and Lawrence, P. (1989). Distribution of the wingless gene product in Drosophila embryos: A protein involved in cell-cell communication. Cell 59, 739-749.

White, R. and Wilcox, M. (1985). Distribution of Ultrabithorax proteins in Drosophila. EMBO J. 4, 2035-2043.

Wilkins, A. and Gubb, D. (1991). Pattern formation in the embryo and imaginal discs of Drosophila: What are the links? Dev. Biol. 145, 1-12.

Wirz, J., Fessler, L. and Gehring, W. (1986). Localization of the Antennapedia protein in Drosophila embryos and imaginal discs. EMBO J. 5, 3327-3334.

(Accepted 4 September 1992) 
